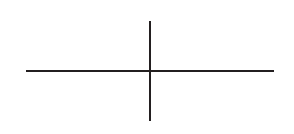

Tohoku Math. J.

57 (2005), 261-271

\title{
$f$-STRUCTURES ON THE CLASSICAL FLAG MANIFOLD WHICH ADMIT (1,2)-SYMPLECTIC METRICS
}

\author{
Nir Cohen, ${ }^{*}$ Caio J. C. Negreiros, Marlio Paredes, ${ }^{\dagger}$ \\ SOFIA PINZÓN ${ }^{\ddagger}$ AND Luiz A. B. SAN MARTIN ${ }^{\S}$
}

(Received August 22, 2003, revised April 16, 2004)

\begin{abstract}
We characterize the invariant $f$-structures $\mathcal{F}$ on the classical maximal flag manifold $\boldsymbol{F}(n)$ which admit $(1,2)$-symplectic metrics. This provides a sufficient condition for the existence of $\mathcal{F}$-harmonic maps from any cosymplectic Riemannian manifold onto $\boldsymbol{F}(n)$. In the special case of almost complex structures, our analysis extends and unifies two previous approaches: a paper of Brouwer in 1980 on locally transitive digraphs, involving unpublished work by Cameron; and work by Mo, Paredes, Negreiros, Cohen and San Martin on conefree digraphs. We also discuss the construction of $(1,2)$-symplectic metrics and calculate their dimension. Our approach is graph theoretic.
\end{abstract}

1. Introduction. Gray [9] and Lichnerowicz [12] were among the first to observe the relevance of $(1,2)$-symplectic structures, not necessarily invariant or Kähler, in Hermitian geometry and harmonic maps, respectively. Originally, almost complex structures were considered, but there is interest in studying the more general case of $f$-structures [24], [1].

Here we consider the special case of the maximal flag manifold $\boldsymbol{F}(n)$ associated with $\mathfrak{s l}(n, \boldsymbol{C})$, endowed with an invariant $f$-structure $\mathcal{F}$. Following Burstall and Salamon [5] and Black [1], there is interest in analyzing the conditions under which $\mathcal{F}$ admits an invariant metric $d s^{2}$ on $\boldsymbol{F}(n)$ which is (1,2)-symplectic, i.e., $(d \mathcal{F})^{(1,1)} \equiv 0$ (see [19] or Section 2 below). We call such a structure $(1,2)$-admissible. In this paper we characterize the invariant structures of this type.

The pair $(\boldsymbol{F}(n), \mathcal{F})$ defines in a natural way a digraph (oriented graph) $\mathcal{G}=(V, E)$, while the metric $d s^{2}$ provides a weighting $\lambda_{e}>0, e \in E$. The (1,2)-symplectic conditions constitute a simple system of linear homogeneous restrictions on the weights $\lambda_{e}$. The issue is, therefore, finding a necessary and sufficient condition for the consistency of this system.

A special case of interest is when the invariant structure $\mathcal{F}$ is almost Hermitian. Here, the digraph $\mathcal{G}$ is complete, i.e., a tournament digraph. It was suggested by Mo and Negreiros [13] that $\mathcal{F}$ admits $(1,2)$-symplectic metrics if and only if $\mathcal{G}$ is cone-free, namely omits certain subgraphs. This has been verified in some cases by Paredes [16], [17], and demonstrated in the general case by Cohen, Negreiros and San Martin [6], [7]. Up to permutation, the incidence

2000 Mathematics Subject Classification. Primary 53C55; Secondary 22F30, 17B45, 05C20.

Key words and phrases. Flag manifolds, (1,2)-symplectic structures, directed graphs.

* Supported by CNPq, grant number 300019/96-3.

${ }^{\dagger}$ Supported by COLCIENCIAS, contract number 240-2001.

¥ Supported by CNPq.

$\S$ Supported by CNPq, grant number 301060/94-0. 


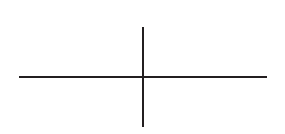

matrix of such a digraph has a stair-shaped form, and remains so under the cyclic shift in $n$ indices [6], [7].

Another class of digraphs, also preserved by the cyclic shift, called locally transitive digraphs, has been studied earlier in Brouwer's paper [3]. We show that the cone-free and locally-transitive conditions are, in fact, equivalent and define the same family of digraphs. We thank Brendan McKay (ANU Canberra, Australia) for bringing [3] to our attention.

We extend the definition of local transitivity from complete digraphs to non-complete digraphs, and in our main result (Theorem 5.1) we establish a one to one correspondence between $f$-structures and digraphs, such that the $(1,2)$-admissible structures correspond precisely to the locally transitive digraphs. This way, given a triple $\left(\boldsymbol{F}(n), \mathcal{F}, d s^{2}\right)$, one can often verify, based on the structure $\mathcal{F}$ alone, that $d s^{2}$ is not $(1,2)$-symplectic. For more details see [18].

Brouwer [3] gave a number-theoretic formula for the number of non-equivalent complete locally transitive digraphs with $n$ vertices. Using the above observation, it can be seen that the same formula describes the number of $(1,2)$-admissible invariant almost complex structures on $\boldsymbol{F}(n)$ up to a natural equivalence. The same problem for $f$-structures remains open.

Another interesting problem is the description of the full set of $(1,2)$-symplectic metrics associated with an $f$-structure. In the case of almost complex structures, this was done in [6] and [7]. We discuss this problem for the general case of $f$-structures on $\boldsymbol{F}(n)$ but do not solve it completely.

It would also be interesting to connect the results obtained here with the existence and classification of harmonic maps into $\boldsymbol{F}(n)$.

2. Geometric flag manifolds and $f$-structures. Consider the classical maximal flag manifold $\boldsymbol{F}(n)=U(n) / T$, with $n \geq 2$, where $U(n)$ is the unitary group and $T$ is a maximal torus in $U(n)$. We shall follow the definitions and notation of [7] and [20]. In particular, $\mathfrak{u}(n)$ is the Lie algebra of $U(n)$ and $\mathfrak{s l}(n, \boldsymbol{C})$ is its complexification. If $b=T$ stands for the origin in $\boldsymbol{F}(n)$, the tangent space at $b$ identifies naturally with the subspace $\mathfrak{q} \subset \mathfrak{u}(n)$ spanned by $A_{j k}=E_{j k}-E_{k j}$ and $S_{j k}=i\left(E_{j k}+E_{k j}\right)$. Here $E_{j k}$ is the matrix with 1 in entry $j k$ and zeros otherwise.

By classical theory an invariant metric $d s^{2}$ on $\boldsymbol{F}(n)$ can be identified with an inner product in $\mathfrak{q}$ of the form $(X, Y)_{\Lambda}=-\langle\Lambda(X), Y\rangle$, with $\Lambda: \mathfrak{q} \rightarrow \mathfrak{q}$ positive definite with respect to the Cartan-Killing form $\langle X, Y\rangle=\operatorname{tr}(\operatorname{ad}(X) \operatorname{ad}(Y))$ where $\operatorname{ad}(\cdot)$ stands for the adjoint representation of $\mathfrak{u}(n)$. The inner product $(\cdot, \cdot)_{\Lambda}$ admits a natural extension to a bilinear symmetric form on $\mathfrak{q}^{C}$. We use the same notation $(\cdot, \cdot)_{\Lambda}$ for this form, as well as for the correspondent complexified form $\Lambda$. The metric $(\cdot, \cdot)_{\Lambda}$ is invariant if and only if $E_{j k}, 1 \leq j, k \leq n$ are the eigenvectors of $\Lambda$, that is,

$$
\Lambda\left(E_{j k}\right)=\lambda_{j k} E_{j k}
$$

with $\lambda_{j k}=\lambda_{k j}>0$ for $1 \leq j, k \leq n$. 


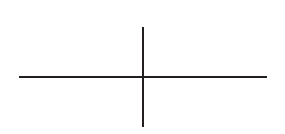

For the real space $\mathfrak{q}$, the elements of the canonical basis $A_{j k}, S_{j k}$, with $1 \leq j<k \leq n$, are eigenvectors for the same eigenvalue $\lambda_{j k}$. We denote by $d s_{\Lambda}^{2}$ the $U$-invariant metric associated with $\Lambda$. In what follows we will use $\Lambda$ as synonymous for $d s_{\Lambda}^{2}$. As a special case, the Cartan-Killing inner product $\langle X, Y\rangle$ is induced by the Cartan-Killing metric corresponding to $\lambda_{j k}=1$ for all $1 \leq j, k \leq n$.

An $f$-structure (see [24]) on $\boldsymbol{F}(n)$ is a section $\mathcal{F}$ of the bundle $\operatorname{End}(T \boldsymbol{F}(n))$ which satisfies $\mathcal{F}^{3}+\mathcal{F}=0$. A $U$-invariant $f$-structure in $\boldsymbol{F}(n)$ is completely determined by an endomorphism $\mathcal{F}: \mathfrak{q} \rightarrow \mathfrak{q}$, satisfying $\mathcal{F}^{3}+\mathcal{F}=0$, which commutes with the adjoint action of $T$. $\mathcal{F}$ extends uniquely to an endomorphism $\mathcal{F}: \mathfrak{q}^{C} \rightarrow \mathfrak{q}^{C}$ which is diagonalizable with eigenvalues $i, 0,-i$ (we denote by $i$ the complex unit $\sqrt{-1}$ ). Let $\mathfrak{q}^{+}, \mathfrak{q}^{0}, \mathfrak{q}^{-}$be the correspondent eigenspaces, we have $\mathfrak{q}^{C}=\mathfrak{q}^{+}+\mathfrak{q}^{0}+\mathfrak{q}^{-}$with $\overline{\mathfrak{q}^{+}}=\mathfrak{q}^{-}$.

The $U$-invariance of $\mathcal{F}$ guarantees that the basic vectors $E_{j k}$ are eigenvectors for $\mathcal{F}$. Thus $\mathcal{F}$ is determined uniquely by the values $\varepsilon_{j k} \in\{0, \pm 1\}$, given by $\mathcal{F}\left(E_{j k}\right)=i \varepsilon_{j k} E_{j k}$, and satisfying $\varepsilon_{k j}=-\varepsilon_{j k}$. In the sequel we allow an abuse of notation and identify the invariant $f$-structure $\mathcal{F}$ on $\boldsymbol{F}(n)$ with $\left\{\varepsilon_{j k}: 1 \leq j, k \leq n\right\}$. In particular an invariant $f$ structure with $\mathcal{F}^{2}=-1$ is an invariant almost complex structure. In our invariant context this amounts to $\varepsilon_{j k} \neq 0$ for all $j, k$.

We say that $\left(\boldsymbol{F}(n), \mathcal{F}, d s^{2}\right)$ is $(1,2)$-symplectic if $(d \mathcal{F})^{(1,1)} \equiv 0$, that is, $(\nabla \mathcal{F})(X, Y)=0$ whenever $X \in \mathfrak{q}^{+}$and $Y \in \mathfrak{q}^{-}$, where $d$ is the exterior derivative. When $\mathcal{F}$ is an almost complex structure, this definition is equivalent to $(d \Omega)^{(1,2)}=0$, where $\Omega$ is the Kähler form [11].

We say that $(\boldsymbol{F}(n), \mathcal{F})$ is $(1,2)$-admissible if there exists a Hermitian metric $d s^{2}$ such that $\left(\boldsymbol{F}(n), \mathcal{F}, d s^{2}\right)$ is $(1,2)$-symplectic.

The relevance of the study of $f$-structures in relation to flag manifolds is highlighted by a theorem of Black [1], which gives a sufficient condition on $\mathcal{F}$ so that a map $\phi:\left(M^{2}, g, J\right) \rightarrow$ $\left(\boldsymbol{F}(n), d s^{2}, \mathcal{F}\right)$ be harmonic with respect to (the given metric $g$ and) every invariant metric $d s^{2}$. Using the Toda field equations and Black's theorem, harmonic tori in symmetric spaces, such as projective or Grassmannian spaces, have been studied (see for example [2], [4]).

3. Graph theoretic description of $(\boldsymbol{F}(n), \mathcal{F}, \Lambda)$. A digraph is a finite oriented graph $\mathcal{G}=(V, E)$. If $v, w \in V$, then an arrow $v \rightarrow w$ indicates that $v w \in E$, while $v \leftrightarrow w$ indicates either $v w \in E$ or $w v \in E$. Furthermore, we define the $v$-loser and $v$-winner sets to be

$$
\mathcal{G}_{L}(v)=\{w \in V ; w v \in E\}, \quad \mathcal{G}_{W}(v)=\{w \in V ; v w \in E\},
$$

considered as subdigraphs of $\mathcal{G}$. This is analogous to the concept of neighbor set used in nonoriented graphs. Finally, we say that $v$ is a winner (resp. loser) in $\mathcal{G}$ if $\mathcal{G}_{L}(v)$ (resp. $\mathcal{G}_{W}(v)$ ) equals to $V \backslash\{v\}$.

The invariant $f$-structures on $\boldsymbol{F}(n)$ are in 1:1 correspondence with digraphs $\mathcal{G}=(V, E)$. The correspondence is given by associating with the $f$-structure $\mathcal{F}\left(E_{j k}\right)=i \varepsilon_{j k} E_{j k}$ a digraph $\mathcal{G}$ whose vertices are $\{1, \ldots, n\}$ and whose arrows are given by the following rules: For $j<k$ 

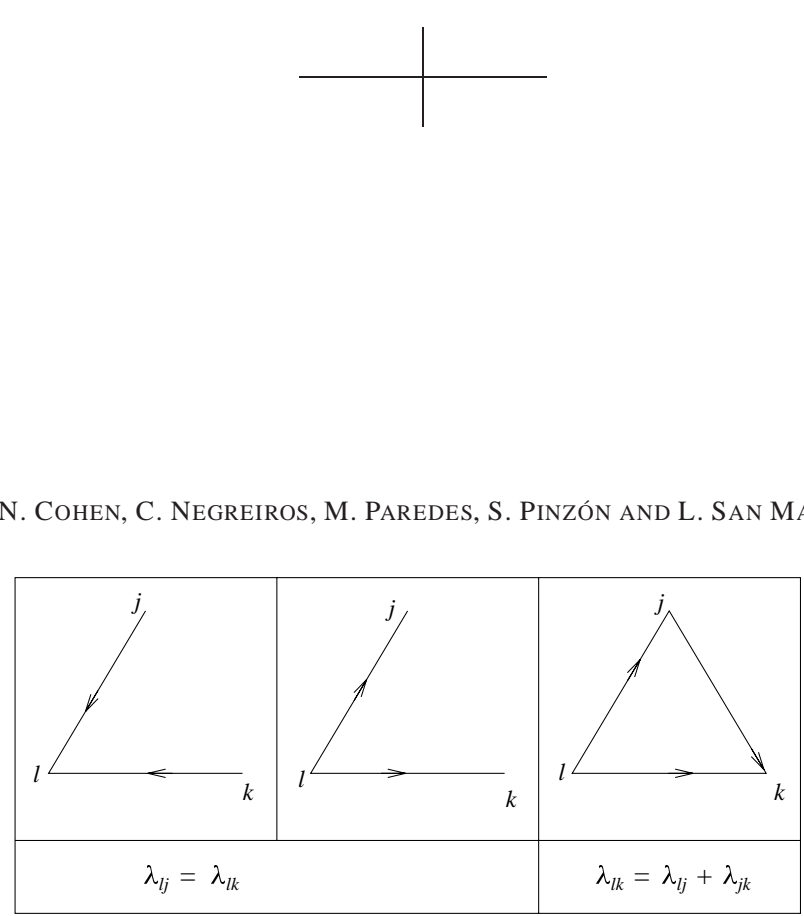

FIGURE 1. 3-vertex subdigraphs associated with local transitivity.

$$
\begin{aligned}
& j \rightarrow k \Longleftrightarrow \varepsilon_{j k}=1, \\
& j \leftarrow k \Longleftrightarrow \varepsilon_{j k}=-1, \\
& j \leftrightarrow k \Longleftrightarrow \varepsilon_{j k}=0 .
\end{aligned}
$$

Similarly, through the matrix $\Lambda=\left\{\lambda_{j k}\right\}$ we may identify an invariant metric $d s^{2}$ on $(\boldsymbol{F}(n), \mathcal{F})$ with a positive weighting on the edge set $E$ of the digraph. Note that if $\varepsilon_{j k}=0$, the weight $\lambda_{j k}$ may be ignored, since $j k \notin E$. According to [1], the (1,2)-symplecticity conditions imposed by $\varepsilon$ on the metric $\Lambda$ amount to the following three rules:

$$
\begin{aligned}
& \text { If } \quad j \rightarrow l, k \rightarrow l, j \leftrightarrow k, \quad \text { then } \quad \lambda_{j l}=\lambda_{k l} ; \\
& \text { If } \quad l \rightarrow j, \quad l \rightarrow k, j \leftrightarrow k, \quad \text { then } \lambda_{l j}=\lambda_{l k} ; \\
& \text { If } \quad l \rightarrow j, j \rightarrow k, l \rightarrow k, \quad \text { then } \quad \lambda_{l k}=\lambda_{l j}+\lambda_{j k} .
\end{aligned}
$$

These restrictions apply to any 3-vertex subdigraph of $\mathcal{G}$ of the types given in Figure 1 .

As stated in the Introduction, our main problem is the characterization of $f$-structures which admit (1,2)-symplectic metrics. In graph-theoretic terms, we wish to characterize the digraphs $\mathcal{G}=(V, E)$ which admit positive weights $\Lambda$ which satisfy properties (1) trough (3). It is this version of the problem which we shall consider in the rest of the paper.

The following definitions will be crucial for our main result.

DEFINITION 3.1. A digraph $\mathcal{G}:=(V, E)$ is called: (i) transitive if the relation " $\rightarrow$ " is transitive (i.e., for $i, j, k \in V, i \rightarrow j \rightarrow k$ implies $i \rightarrow k$ ), (ii) relatively connected if for all $i, j, k \in V i \rightarrow j$ implies $i \leftrightarrow k$ or $j \leftrightarrow k$.

Transitivity for complete digraphs may be characterized by the absence of cycles, and the incidence matrix of such digraphs is permutation-similar to the canonical matrix $\varepsilon_{j k}=1$ $(j<k)$ [14]. We shall be more interested in the following local version of this property.

DEFINITION 3.2. We call the digraph $\mathcal{G}=(V, E)$ locally transitive (in short, $\mathrm{LT}$ ) if for all $v \in V$ each of the subdigraphs $\mathcal{G}_{L}(v)$ and $\mathcal{G}_{W}(v)$ is transitive and relatively connected.

The LT and non-LT 4-digraphs are given in Figures 2 and 3. 

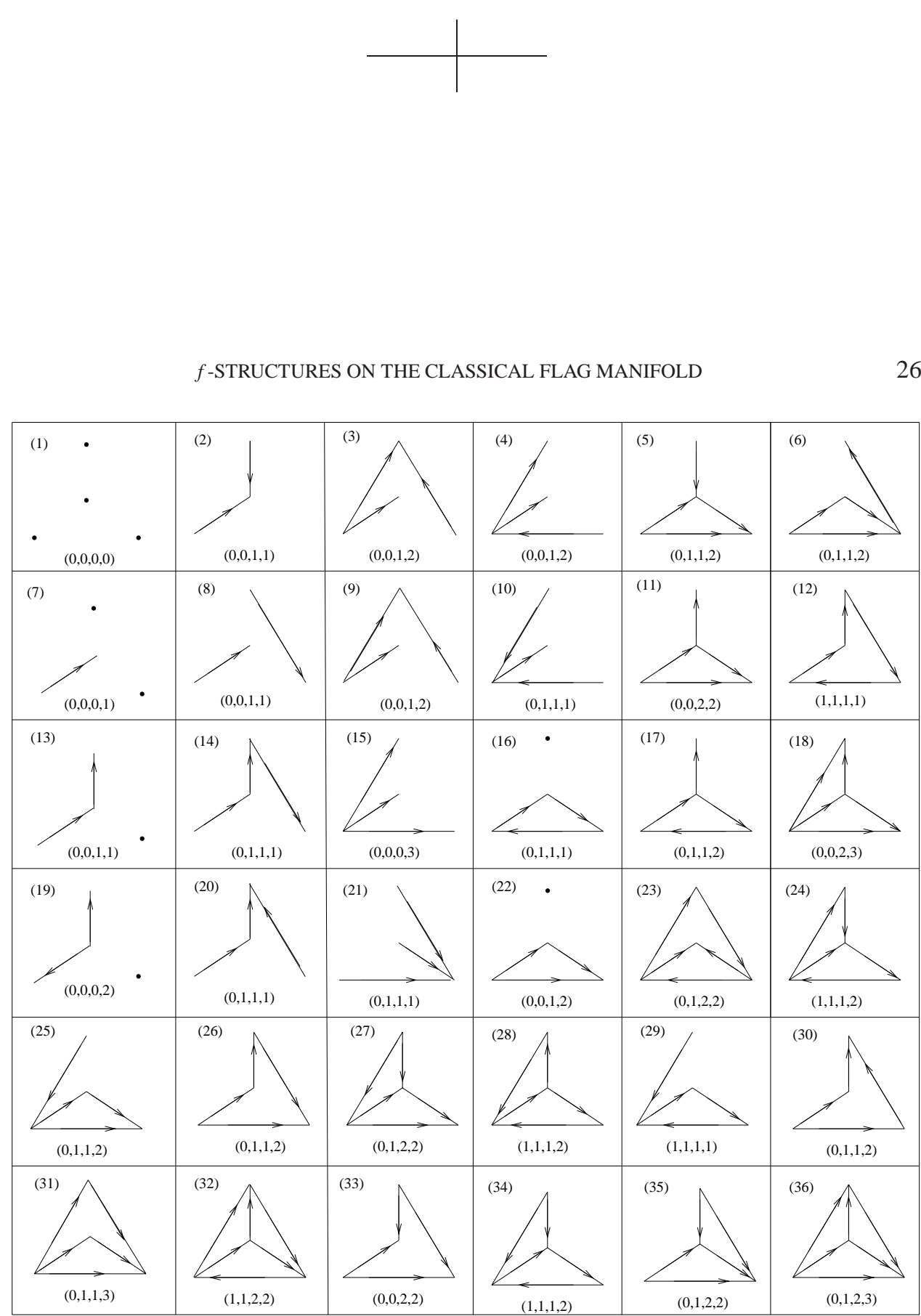

FIGURE 2. The thirty six 4-vertex digraphs which are LT.

Several remarks are in order:

(i) Local transitivity means that the digraphs $\mathcal{G}_{L}(v)$ and $\mathcal{G}_{W}(v)$ omit certain 3-vertex subdigraphs, namely the ones whose edges form a non-empty subset of a 3-cycle (compare with Figures 2 and 3).

(ii) In case $\mathcal{G}$ is complete, local transitivity implies that both $\mathcal{G}_{W}(v)$ and $\mathcal{G}_{L}(v)$ are (complete and) transitive. This way we recover the original definition introduced for complete digraphs by P. J. Cameron (unpublished) and discussed in [3].

(iii) If $\max \left\{\left|\mathcal{G}_{W}(v)\right|,\left|\mathcal{G}_{L}(v)\right|\right\} \leq 2$ for all $v \in V$ then $\mathcal{G}$ is LT.

(iv) All the digraphs of size $\leq 3$ are LT. As to $n=4$, simple analysis shows that up to digraph isomorphism there exist 42 digraphs with 4 vertices, six of which are not LT. As seen 

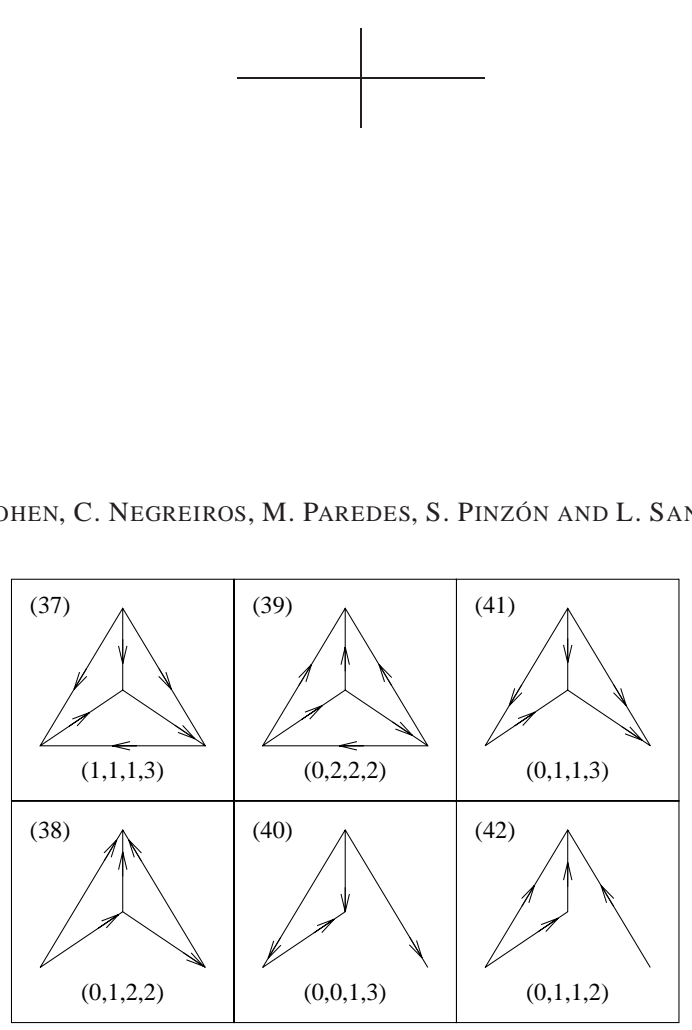

FIGURE 3. The six 4-vertex digraphs which are not LT.

from Figure 3, a non-LT 4-vertex digraph must have a winner or a loser, but not both. If $v$ is the winner/loser, then $V_{W}(v)$ (resp. $\left.V_{L}(v)\right)$ is a non-trivial subdigraph of a 3-cycle (see [18]).

LEMMA 3.3. Let $\mathcal{G}=(V, E)$ with $|V| \geq 4$. $\mathcal{G}$ is $L T$ if and only if every 4-vertex subdigraph of $\mathcal{G}$ is $L T$.

PROOF. If $\mathcal{G}$ is LT then any subdigraph of $\mathcal{G}$, including all the 4 -vertex subdigraphs, is LT. It remains to show the converse direction. Assuming $\mathcal{G}$ is not LT, we have two cases, both leading to the existence of a non-LT 4-subdigraph, completing the proof.

Case 1. There exists $v \in V$ such that one of the sets $\mathcal{G}_{L}(v), \mathcal{G}_{W}(v)$ is not transitive. Namely, in this set there exist $j, k$ and $l$ such that $j k, k l \in E$, but $j l \notin E$. It can be checked against Figure 3 that whether $l j \in E$ or not, the subdigraph of $\mathcal{G}$ supported on $\{v, j, k, l\}$ is not LT.

Case 2. There exists $v \in V$ such that one of the sets $\mathcal{G}_{L}(v), \mathcal{G}_{W}(v)$ is neither trivial nor relatively connected. Namely, in this set there exist $j, k$ and $l$ such that $j k \in E$, but $j l, k l, l j, l k \notin E$. Here the subdigraph supported on $\{v, j, k, l\}$ is also not LT.

The case of almost complex structures, which correspond to complete digraphs, is of special interest. Exactly two of the six 4-vertex digraphs in Figure 3 are complete: those which contain a winner/loser and a 3-cycle. In [13] these two digraphs were called "cones", and in [6], [7] a complete digraph $\mathcal{G}$ which omitted them was called "cone-free". Lemma 3.3 states, therefore, that $\mathcal{G}$ is LT if and only if it is cone-free. As a result, the two families of complete digraphs studied separately in [6], [7], [13], [17] (coneless tournaments) and in [3] (LT tournaments) are shown to be one and the same.

4. Completely non-transitive structures. An $f$-structure $\mathcal{F}$ on $\boldsymbol{F}(n)$ will be called completely non-transitive if the Cartan-Killing metric $\left(\lambda_{i j} \equiv 1\right.$, for all $\left.1 \leq j, k \leq n\right)$ is $(1,2)$-symplectic with respect to $\mathcal{F}$. Structures of this type have a simple graph theoretic description, and all the $(1,2)$-symplectic metrics relative to such a structure can be described. First we characterize the corresponding digraphs. 


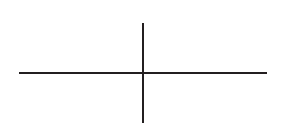

Definition 4.1. (i) A transitive triangle is a transitive digraph $\mathcal{G}_{t}=\left(V_{t}, E_{t}\right)$ with $\left|V_{t}\right|=3$. Assuming $V_{t}=\{u, v, w\}$ and $E_{t}=\{u v, v w, u w\}$, we shall refer to $u v, v w$ as sides and to $u w$ as a base. (ii) We shall call a digraph $\mathcal{G}^{\prime}=\left(V^{\prime}, E^{\prime}\right)$ completely non-transitive if it does not contain any transitive triangle.

LEMMA 4.2. An invariant $f$-structure $\mathcal{F}$ on $\boldsymbol{F}(n)$ is completely non-transitive if and only if the associated digraph $\mathcal{G}$ is completely non-transitive.

PROOF. If $\mathcal{G}$ is completely non-transitive then, due to the absence of transitive triangles, system (1-3) has no identities of type (3) and hence the Cartan-Killing metric $\lambda \equiv 1$, which automatically satisfies (1-2), is (1,2)-symplectic. Conversely, if the Cartan-Killing metric is $(1,2)$-symplectic, then (3) cannot occur, hence $\mathcal{G}$ contains no transitive triangles.

A completely non-transitive digraph is $L T$. Indeed, the sets $\mathcal{G}_{W}^{\prime}(v)$ and $\mathcal{G}_{L}^{\prime}(v)$ contain no edges (also, the digraph is cone-free, since every cone contains a transitive triangle). At the same time, the corresponding $f$-structure is $(1,2)$-admissible due to Lemma 4.2. In the special case of complete digraphs, Lemma 4.2 implies that the Cartan-Killing metric is $(1,2)$ symplectic only if $|V|<3$, as observed in [6].

Define the following equivalence relation between edges in $E^{\prime}: e \sim e^{\prime}$ if for some $v, v^{\prime}, u \in V$ we have either $e=v u$ and $e^{\prime}=v^{\prime} u$, or $e=u v$ and $e^{\prime}=u v^{\prime}$. A metric on $\mathcal{G}^{\prime}$ is $(1,2)$-symplectic if and only if it is constant on every equivalence class in $E^{\prime}$. Thus, the dimension of the cone of invariant $(1,2)$-symplectic metrics is equal to $\beta$, the number of equivalence classes in $E^{\prime}$.

How can $\beta$ be calculated from $\mathcal{G}^{\prime}$ directly? We do not know the answer, but a promising observation is that $\beta$ is the number of connected components in a "spanning forest" for $\mathcal{G}^{\prime}$, assuming every vertex in the forest is a winner or a loser.

In studying (1,2)-symplectic invariant metrics on $(\boldsymbol{F}(n), \mathcal{F}, \Lambda)$ associated to a general LT digraph $\mathcal{G}$, our approach consists in the reduction of $\mathcal{G}$ to an associated completely nontransitive subdigraph $\mathcal{G}^{\prime}$ with the same vertex set, based on the following "edge deletion lemma".

LEMMA 4.3. Let $\mathcal{G}=(V, E)$ be a LT digraph which is not completely non-transitive (see Definition 4.1). Then $E$ contains an edge $e$ which is a base but not a side. In this case, the subdigraph $\tilde{\mathcal{G}}:=(V, E \backslash\{e\})$ is LT.

Proof. Let $\mathcal{G}_{*}=\left(V_{*}, E_{*}\right)$ be a maximal subdigraph of $\mathcal{G}$ which is complete and transitive, and $\left|V_{*}\right| \geq 3$. The assumption guarantees the existence of at least one such subdigraph. Then $\mathcal{G}_{*}$ has a single base $e \in E_{*}$ which is not a side, namely the arrow $e$ which connects the winner and loser in $\mathcal{G}_{*}$.

The edge $e$ is therefore a base in $\mathcal{G}$. We claim that $e$ cannot be a side in some transitive triangle in $\mathcal{G}$. Assume to the contrary that such a triangle $\mathcal{G}_{t}=\left(V_{t}, E_{t}\right)$ does exist. Note that $V_{t} \not \subset V_{*}$, since $e$ is not a side in $\mathcal{G}_{*}$. Therefore, the subdigraph $\mathcal{G}^{*}$ of $\mathcal{G}$ supported on $V_{*} \cup V_{t}$ strictly contains $\mathcal{G}^{*}$. Local transitivity of $\mathcal{G}$ implies that $\mathcal{G}^{*}$ is again complete and transitive, contradicting the maximality of $\mathcal{G}_{*}$. 


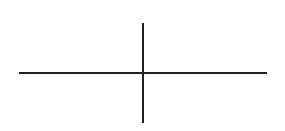

Next we show that $\tilde{\mathcal{G}}=(V, E \backslash\{e\})$ is LT. By Lemma 3.3 it suffices to show that every 4-subdigraph $\hat{\mathcal{G}}=(\hat{V}, \hat{E}) \subset \tilde{\mathcal{G}}$ is LT. Assume that $e=u w, u, w \in V$. If $\{u, w\} \not \subset \hat{V}$, then $\hat{\mathcal{G}}$ is a subdigraph of $\mathcal{G}$, and hence is LT by Lemma 3.3. Otherwise, if $\{u, w\} \subset \hat{V}$, suppose that $\hat{\mathcal{G}}$ is not LT. According to a remark (i) made in Section 3, $\hat{\mathcal{G}}$ contains a winner or a loser, say $v$. It follow from $e \notin \hat{E}$ that $v \neq u, w$. Whether $v$ is a winner or a loser, $e$ is a side in the transitive triangle $\{u, v, w\}$ in $\mathcal{G}$, which is impossible by the first part of the Lemma.

We close this section with several remarks (see [18]):

(i) The completely non-transitive digraph $\mathcal{G}^{\prime}$ obtained by edge deletion from $\mathcal{G}$ does not depend on the order of the edges deleted.

(ii) A completely non-transitive digraph $\mathcal{G}^{\prime}$ can be the outcome of a non-void edge deletion of a LT digraph if and only if the union of all the subdigraphs of $\mathcal{G}^{\prime}$ of type $\{u v, v w, z w\}$ and $\{w z, w v, v u\}$ does not contain every subdigraph of type $\{u v, v w\}$.

(iii) It seems that a completely non-transitive digraph is the result of edge deletion of a transitive digraph if and only if for all vertex $v$ which is not a winner or a loser, $\left|\mathcal{G}_{W}(v)\right|=$ $\left|\mathcal{G}_{L}(v)\right|=1$.

5. Characterization of $(\mathbf{1 , 2})$-admissible $f$-structures. We will now state the central result in our paper. The proof of this result is combinatorial and relies heavily on the results obtained in the preceding sections.

THEOREM 5.1. Let $\mathcal{F}$ be an invariant $f$-structure on $\boldsymbol{F}(n), n \geq 2$. $(\boldsymbol{F}(n), \mathcal{F})$ admits invariant $(1,2)$-symplectic metrics if and only if $\mathcal{F}$ is $L T$.

We will prove the following clearly equivalent statement:

Lemma 5.2. The digraph $\mathcal{G}=(V, E),|V| \geq 2$ admits (1,2)-symplectic invariant metrics if and only if it is $L T$.

PROOF. For $n<4, \mathcal{G}$ is always LT, and verification of the lemma is an easy exercise. For $n=4$, verification is easy, based on the digraphs in Figure 3. So, assume $n>4$.

If $\mathcal{G}$ admits $(1,2)$-symplectic invariant metrics, then by restriction every 4-vertex subdigraph of $\mathcal{G}$ admits $(1,2)$-symplectic metrics, and hence (as just observed) is LT. But then by Lemma $3.3, \mathcal{G}$ is LT.

Conversely, assume that $\mathcal{G}$ is LT. We argue by induction. If $\mathcal{G}$ is completely non-transitive, then the existence of $(1,2)$-symplectic metrics was guaranteed in the previous section. Otherwise, by Lemma 4.3, we may delete an edge $e$ from $\mathcal{G}=(V, E)$, obtaining another LT digraph $\tilde{\mathcal{G}}=(V, \tilde{E})$. By the induction argument, $\tilde{\mathcal{G}}$ has $(1,2)$-symplectic metrics. We extend each such metric to a metric on $\mathcal{G}$ by defining $\lambda_{e}=\lambda_{e^{\prime}}+\lambda_{e^{\prime \prime}}$, where $e^{\prime}$ and $e^{\prime \prime}$ are the sides corresponding to the base $e$. This is the only extension for which $\Lambda$ is $(1,2)$-symplectic on the triangle in question, and hence the only extension which might be $(1,2)$-symplectic for the whole digraph. We want to show that, in fact, it is.

Step 1. We show that the extension is well-defined. Namely, assume that $e=u w$ is simultaneously a base for two transitive triangles, say $\{u v, v w, u w\}$ and $\{u z, z w, u w\}$ with 


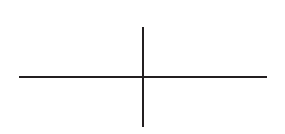

$\{u, v, w, z\} \subset V$. We need to show that a priori

$$
\lambda_{u z}+\lambda_{z w}=\lambda_{u v}+\lambda_{v w} .
$$

There are two cases to consider. If $v \leftrightarrow z$, we may assume for definiteness that $v \rightarrow z$. In this case, by (3) we have a priori $\lambda_{v z}=\lambda_{u z}-\lambda_{u v}$ and $\lambda_{v z}=\lambda_{v w}-\lambda_{z w}$, implying (4). Otherwise, if $v \nLeftarrow z$, by (1-2) we have a priori $\lambda_{u v}=\lambda_{u z} \lambda_{v w}=\lambda_{z w}$, again implying (4).

Step 2. We show that the extended metric is $(1,2)$-symplectic. Every conflict within the constraint system (1) through (3) should involve the deleted edge $e$, since $\tilde{\mathcal{G}}$ is assumed to satisfy these restrictions. By Lemma 4.3, $e$ is not a side in $\mathcal{G}$, and hence any conflict with (3) is of the type already discussed in Step 1.

A conflict with (1) implies that $\lambda_{e} \neq \lambda_{e}^{\prime}$ where, say, $e=u w$ and $e^{\prime}=t w$. This can occur only if $t \nLeftarrow u$. Now it can be easily seen that independently of the relation between $v$ and $t, \mathcal{G}$ and $\tilde{\mathcal{G}}$ cannot both be LT, since one of the two contains one of the non-LT digraphs of Figure 3. This is a contradiction to our assumptions.

A conflict with (2) leads to a similar contradiction, and so the proof is complete.

Assume that the LT digraph $\mathcal{G}$ is reduced, via edge deletion, to a completely non-transitive digraph $\mathcal{G}^{\prime}$. Theorem 5.1 shows that every $(1,2)$-symplectic metric on $\mathcal{G}^{\prime}$ extends uniquely to a $(1,2)$-symplectic metric on $\mathcal{G}$. Thus, the dimension of the cone of $(1,2)$-symplectic metrics is equal in both digraphs (in the previous section it was denoted by $\beta$ ). It is not clear how to calculate $\beta$ directly from the original digraph $\mathcal{G}$. One approach is to represent (1 through 3 ) as a homogeneous linear system and calculate the dimension of its kernel.

The classification of (essentially different) (1,2)-admissible invariant structures on $\boldsymbol{F}(n)$ is reduced, via Theorem 5.1, to the classification of (permutationally different) LT digraphs. Brouwer [3] applied a nice counting argument in order to enumerate the complete LT digraphs with $n$ vertices. On first reading it appears that his method is not adequate for the enumeration of all the LT digraphs with $n$ vertices. One possible attack on the problem would be to enumerate first the completely non-transitive ones, and then to figure out how many LT digraphs edge-delete into a given completely non-transitive digraph.

\section{REFERENCES}

[1] M. BlaCK, Harmonic maps into homogeneous spaces, Pitman Research Notes in Mathematics Series 255, Longman Scientific \& Technical, Harlow, 1991.

[2 2 J. Bolton And L. M. Woodward, Minimal surfaces and the affine Toda field model, J. Reine Angew. Math. 459 (1995), 119-150.

[ 3 ] A. E. BROUWER, The enumeration of locally transitive tournaments, Afdeling Zuivere Wiskunde [Department of Pure Mathematics] 138, Mathematisch Centrum, Amsterdam, 1980.

[ 4 ] F. E. Burstall, D. Ferus, F. Pedit and U. Pinkall, Harmonic tori in symmetric spaces and commutative Hamiltonian systems on loop algebras, Ann. of Math. (2) 138 (1993), 173-212.

[ 5 ] F. E. Burstall And S. Salamon, Tournaments, flags and harmonic maps, Math Ann. 277 (1987), 249265. 


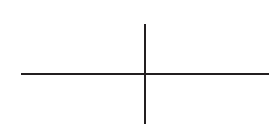

[6] N. Cohen, C. J. C. Negreiros And L. A. B. San Martin, Decription of (1,2)-symplectic metrics on flag manifolds, Global differential geometry: the mathematiocal legacy of Alfred Gray (Bilbao, 2000), 300-304, Contemp. Math. 288, Amer. Math. Soc., Providence, R. I., 2001.

[ 7 ] N. Cohen, C. J. C. Negreiros and L. A. B. San Martin, (1,2)-Symplectic metrics, flag manifolds and tournaments, Bull. London Math. Soc. 34 (2002), 641-649.

[ 8 ] N. Cohen, C. J. C. Negreiros and L. A. B. San Martin, A rank-three condition for invariant $(1,2)-$ symplectic almost Hermitian structures on flag manifolds, Bull. Braz. Math. Soc. (N.S.) 33(1) (2002), 49-73.

[9] A. Gray, Minimal varieties and almost Hermitian submanifolds, Michigan Math. J. 12 (1965), 273-287.

[10] S. Helgason, Differential geometry, Lie groups and symmetric spaces, Pure Appl. Math. 80, Academic Press, Inc., New York-London, 1978.

[11] S. Kobayashi and K. Nomizu, Foundations of Differential Geometry, Vol. II, Interscience Tracts in Pure and Applied Mathematics 15 Vol. II, Interscience Publishers, John Wiley \& Sons, Inc., New York-LondonSydney, 1969.

[12] A. Lichnerowicz, Applications harmoniques et varietés kähleriennes, 1968/1969 Symposia Mathematica, Vol. III (INDAM, Rome, 1968/69) 341-402, Academic Press, London, 1970.

[13] X. Mo And C. J. C. Negreiros, (1,2)-Symplectic structure on flag manifolds, Tohoku Math. J. (2) 52 (2000), 271-282.

[14] W. Moon, Topics on Tournaments, Holt, Reinhart and Winston, 1968.

[15] C. J. C. Negreiros, Some remarks about harmonic maps into flag manifolds, Indiana Univ. Math. J. 37 (1988), 617-636.

[16] M. Paredes, Aspectos da geometria complexa das variedades bandeira, Ph. D. Thesis, State University of Campinas, 2000.

[17] M. PAREDES, Families of (1,2)-symplectic metrics on flag manifolds, Int. J. Math. Sci. 29 (2002), 651-664,.

[18] S. PinZón, Variedades bandeira, f-structures e métricas (1,2)-simpléticas, Ph. D. Thesis, State University of Campinas, 2003.

[19] J. H. RawnSLey, $f$-Structures, $f$-Twistor spaces and harmonic maps, Geometry seminar "Luigi Bianchi" II-1984, 85-159, Lecture Notes in Math. 1164, Springer, Berlin, 1985.

[20] L. A. B. SAN MARTin AND C. J. C. Negreiros, Invariant almost Hermitian structures on flag manifolds, Adv. Math. 178 (2003), 277-310.

[21] G. WARnER, Harmonic analysis on semi-simple Lie groups I. Grundlehren Math. Wiss. 188, Springer-Verlag, New York-Heidelberg, 1972.

[22] J. A. Wolf and A. Gray, Homogeneous spaces defined by Lie group automorphisms I, J. Differential Geom. 2 (1968), 77-114.

[23] J. A. Wolf And A. Gray, Homogeneous spaces defined by Lie group automorphisms II, J. Differential Geom. 2 (1968) 115-159.

[24] K. YANO, On a structure defined by a tensor field of type $(1,1)$ satisfying $\mathcal{F}^{3}+\mathcal{F}=0$, Tensor (N.S.) 14 (1963), 99-109. 
DePartment of Applied Mathematics IMECC-UNICAMP

CX. Postal 6065, 13083-970

CAMPINAS-SP

BRAZIL

E-mail address: nir@ime.unicamp.br

ESCUELA DE MATEMÁTICAS

UNIVERSIDAD INDUSTRIAL DE SANTANDER

APARTADo AEREo 678

BUCARAMANGA

Colombia

E-mail address:mparedes@uis.edu.co

DEPARTMENT OF MATHEMATICS

IMECC-UNICAMP

Cx. Postal 6065, 13083-970

CAMPINAS-SP

BRAZIL

E-mail address: smartin@ime.unicamp.br
Department of Mathematics IMECC-UNICAMP

CX. Postal 6065, 13083-970

CAMPINAS-SP

BRAZIL

E-mail address: caione@ime.unicamp.br

ESCUELA DE MATEMÁTICAS

UNIVERSIDAD INDUSTRIAL DE SANTANDER

Apartado AEREO 678

BUCARAMANGA

Colombia

E-mail address: sofia@matematics.uis.edu.co 